\title{
ON ASYMPTOTIC PROPERTIES OF SEVERAL CLASSES OF OPERATORS
}

\author{
STEPHEN L. CAMPBELL AND RALPH GELLAR
}

\begin{abstract}
Let $p\left(T, T^{*}\right)$ be a polynomial in $T$ and $T^{*}$ where $T$ is a bounded linear operator on a separable Hilbert space. Let $\Delta=\left\{T \mid p\left(T, T^{*}\right)=0\right\}$. Then $\Delta$ is said to be asymptotic for $p$ if for every $K>0$, there exists an $\varepsilon_{0}>0$ and function $\delta(\varepsilon, K), \lim _{\varepsilon \rightarrow 0} \delta(\varepsilon, K)=0$, such that if $\varepsilon<\varepsilon_{0}$ $\|T\|<K$, and $\left\|p\left(T, T^{*}\right)\right\|<\varepsilon$, then there exists $\hat{T} \in \Delta$ such that $\|T-\hat{T}\|$ $<\delta(\varepsilon, K)$. It is observed that the hermitian, unitary, and isometric operators are asymptotic for the obvious polynomials. It is known that the normals are not asymptotic for $p\left(T, T^{*}\right)=T^{*} T-T T^{*}$. An example gives several negative results including one that says the quasinormals are not asymptotic for $p\left(T, T^{*}\right)=T T^{*} T-T^{*} T^{2}$. It is shown that if $p$ is any polynomial in just one of $T$ or $T^{*}$, then $\Delta$ is asymptotic for $p$.
\end{abstract}

1. Introduction. This paper is concerned with trying to determine whether an operator that almost satisfies the definition of a certain class of operators must be near an operator of that class. To be precise we make the following definition. The underlying space is taken to be a separable Hilbert space $H$.

Definition 1. Let $p\left(T, T^{*}\right)$ be a polynomial in $T$ and $T^{*}$. Let $\Delta=\{T: p(T$, $\left.\left.T^{*}\right)=0\right\}$. We say that $\Delta$ is asymptotic for $p$ if for every $K>0$, there exists an $\varepsilon_{0}>0$ and function $\delta(\varepsilon, K), \lim _{\varepsilon \rightarrow 0} \delta(\varepsilon, K)=0$, such that if $\varepsilon<\varepsilon_{0},\|T\| \leqslant$ $K$, and $\left\|p\left(T, T^{*}\right)\right\|<\varepsilon$ then there exists a $\hat{T} \in \Delta$ such that $\|T-\hat{T}\|<\delta(\varepsilon, K)$.

We shall be primarily interested in determining whether a given $\Delta$ is asymptotic for a given $p$. Our positive results, however, can all be used to get a $\delta(\varepsilon, K)$. The type of problem this paper examines was mentioned by Halmos in a 1973 talk [6]. See also [1], [7].

It should be pointed out that if $H$ is finite-dimensional, then every class defined by a polynomial $p$ is asymptotic. This follows from the compactness of the unit ball in the space of operators on $H$. For normal operators and $p\left(T, T^{*}\right)=T^{*} T-T T^{*}$ see [1].

We will avoid saying "the class $\Delta$ is asymptotic" since a given class may be definable by several different polynomials. An example of a $\Delta$ that is asymptotic for some of these polynomials and not asymptotic for others would be of interest.

$\$ 2$ will cover several easy cases. $\$ 3$ will discuss some negative results. In $\$ 4$,

Presented to the Society, January 29, 1977; received by the editors November 22, 1976.

AMS (MOS) subject classifications (1970). Primary 47A55; Secondary 47B20.

Key words and phrases. Normal, quasinormal, hyponormal, approximation, asymptotic, algebraic.

(1) American Mathematical Society 1977 
we shall show that if $\Delta$ is defined by a polynomial $p$ in just $T$, then $\Delta$ is asymptotic for $p$. Some additional comments will be made in $\S 5$.

2. Selfadjoint and isometric operators. The results of this section are fairly trivial but are included for completeness.

Proposition 1. The class of selfadjoint operators is asymptotic for $p(T$, $\left.T^{*}\right)=T-T^{*}$ and $\delta(\varepsilon, K)$ may be taken as $\delta(\varepsilon, K)=\varepsilon / 2$.

Proof. $\left(T+T^{*}\right) / 2$ is selfadjoint and $\left\|T-\left(T+T^{*}\right) / 2\right\|=\left\|T-T^{*}\right\| / 2$ $=\left\|p\left(T, T^{*}\right)\right\| / 2$.

Proposition 2. The class of isometric operators is asymptotic for $p(T$, $\left.T^{*}\right)=I-T^{*} T$ and $\delta(\varepsilon, K)$ may be taken as $\delta(\varepsilon, K)=\varepsilon$ and $\varepsilon_{0}$ to be 1 .

Proof. If $\left\|I-T^{*} T\right\|<1$, then $T$ is one-to-one and $T=U\left(T^{*} T\right)^{1 / 2}$ with $U$ an isometry. But

$$
\begin{aligned}
\|T-U\| & =\left\|\left(T^{*} T\right)^{1 / 2}-I\right\|=\left\|\left[\left(T^{*} T\right)-I\right]\left[\left(T^{*} T\right)^{1 / 2}+I\right]^{-1}\right\| \\
& \leqslant\left\|p\left(T^{*}, T\right)\right\|\left\|\left[\left(T^{*} T\right)^{1 / 2}+I\right]^{-1}\right\| \leqslant\left\|p\left(T^{*}, T\right)\right\| . \square
\end{aligned}
$$

Similarly, we have:

Proposition 3. The class of coisometric operators is asymptotic for $p(T$, $\left.T^{*}\right)=I-T T^{*}$ and $\delta(\varepsilon, K)$ may be taken as $\varepsilon, \varepsilon_{0}$ as 1 .

There are several different polynomials that give the unitary operators. We mention two.

Proposition 4. The class of unitary operators is asymptotic for $p_{1}\left(T, T^{*}\right)=$ $\left(I-T^{*} T\right)^{2}+\left(I-T T^{*}\right)^{2}$ or $p_{2}\left(T, T^{*}\right)=\left(I-T^{*} T\right)^{2}+\left(T^{*} T-T T^{*}\right)^{2}$. Both $\delta_{1}(\varepsilon, K)$ and $\delta_{2}(\varepsilon, K)$ can be taken as $\sqrt{\varepsilon}$, while $\varepsilon_{0}$ can be taken as 1 in the first case and as $\frac{1}{4}$ in the second.

Proof. It suffices to show that the $U$ in the proof of Proposition 2 is unitary. $U$ is unitary if both $T^{*} T, T T^{*}$ are one-to-one. This follows if $\left\|I-T^{*} T\right\|<1$ and $\left\|I-T T^{*}\right\|<1$ or if $\left\|I-T^{*} T\right\|<\frac{1}{2}$ and $\left\|T^{*} T-T T^{*}\right\|<\frac{1}{2}$.

3. Normal, quasinormal, and hyponormal operators. The property of being normal seems to be very unstable. It is known that $T^{*} T-T T^{*}$ can be compact without $T$ being of the form normal plus compact. Also the class of normal operators is not asymptotic for $p\left(T, T^{*}\right)=T^{*} T-T T^{*}[1]$. A simple example of this is the following:

EXAMPLE 1. Let $T_{n}$ be a weighted unilateral shift with weight sequence $\left\{\alpha_{i}\right\}$ where $\alpha_{i}=\sqrt{i / n}$ for $1 \leqslant i \leqslant n, \alpha_{i}=1$ for $i>n$. Then $\left\|T_{n}\right\|=1$ and $\left\|T_{n}^{*} T_{n}-T_{n} T_{n}^{*}\right\|=1 / n$. Since $T_{n}$ is a compact perturbation of the unilateral shift, $\left\|T_{n}-N\right\| \geqslant 1$ for any normal operator $N[1]$.

The following three results will all follow from the same example. 
THEOREM 1. The class of quasinormal operators is not asymptotic for the polynomial $p\left(T, T^{*}\right)=T T^{*} T-T^{*} T^{2}$.

THEOREM 2. The class of hyponormal operators is not asymptotic for the polynomial inequality $T^{*} T-T T^{*} \geqslant 0$. That is, there exists $\left\{T_{n}\right\},\left\|T_{n}\right\|=1$, $T_{n}^{*} T_{n}-T_{n} T_{n}^{*}+\varepsilon_{n} \geqslant 0, \varepsilon_{n} \rightarrow 0$ but the distance from $T_{n}$ to the hyponormal operators does not go to zero.

TheOREM 3. Let $\Theta=\left\{T \mid\left[T^{*} T, T+T^{*}\right]=0\right\}$. Then $\Theta$ is not asymptotic for

$$
\begin{aligned}
p\left(T, T^{*}\right) & =T^{*} T\left(T+T^{*}\right)-\left(T+T^{*}\right) T^{*} T \\
& =\left(T^{*} T-T T^{*}\right) T-T^{*}\left(T^{*} T-T T^{*}\right) .
\end{aligned}
$$

The class $\Theta$ of Theorem 3 includes the quasinormals but is independent of the hyponormals [5]. The following example will suffice for all three theorems.

EXAMPLE 2. Let $T_{n}$ be a weighted unilateral shift with weight sequence $\left\{\alpha_{i}\right\}$ where $\alpha_{i}=\sqrt{1-i / n}$ for $0 \leqslant i \leqslant n$ and $\alpha_{i}=0$ for $i \geqslant n$. Then $T_{n}^{*} T_{n}$ $T_{n} T_{n}^{*}=\operatorname{Diag}\{1,-1 / n, \ldots,-1 / n, 0, \ldots\}$. Thus $\left\|T_{n} T_{n}^{*} T_{n}-T_{n}^{*} T_{n}^{2}\right\|=$ $1 / n$ and $\left\|T_{n}\right\|=1$. Note that $T_{n}$ is compact and $T_{n}^{*} T_{n}-T_{n} T_{n}^{*} \nrightarrow 0$.

Since quasinormal operators are hyponormal, showing that the $\left\{T_{n}\right\}$ of Example 2 verifies Theorem 2 will also verify Theorem 1 .

Proof of Theorem 2. Let $T_{n}$ be as in Example 2. Suppose that $S_{n}$ is a bounded sequence of hyponormal operators such that $\left\|T_{n}-S_{n}\right\| \rightarrow 0$. Write $S_{n}=H_{n} \oplus N_{n}$ where $H_{n}$ is completely nonnormal and $N_{n}$ is normal. Note that $\left\|H_{n}\right\|$ cannot go to zero since $\left\|T_{n}^{*} T_{n}-T_{n} T_{n}^{*}\right\|$ does not go to zero. But $\left\|T_{n}-S_{n}\right\|_{e}=\left\|S_{n}\right\|_{e} \rightarrow 0$ where $\|\cdot\|_{e}$ is the norm of $S_{n}$ in the Calkin algebra. Thus the essential spectral radius of $S_{n}$, and hence of $H_{n}$, goes to zero. But for completely hyponormal operators, the spectral radius is the essential spectral radius and the spectral radius is the norm. Hence $H_{n} \rightarrow 0$, which is a contradiction.

Theorem 3 may be proved just as was Theorem 2 since if $S_{n} \in \Theta$, then eigenvalues are reducing [3], isolated points of the spectrum are eigenvalues [4], and the spectral radius equals the norm [5].

4. Algebraic operators. The examples of the preceding section show that many of the standard polynomials do not define a class that is asymptotic with respect to that polynomial. In this section we shall show that any class that is defined by a polynomial in just $T$ or $T^{*}$ is asymptotic for that polynomial. We begin with $p(T)=T^{n}$.

LEMMA 1. Suppose that $\left\|T^{n}\right\|<\varepsilon$ for some $n \geqslant 2$ and $\|T\| \leqslant 1$. Let $E$ be the spectral projection for $T^{*} T$ associated with $[0, \varepsilon]$. Then $\left\|[(I-E) T(I-E)]^{n-1}\right\| \leqslant(n-1) \sqrt{\varepsilon}$.

Proof. Define $E$ as in the statement of the lemma. Then $\|T E\| \leqslant \sqrt{\varepsilon}$. But 


$$
\begin{aligned}
& \varepsilon^{2}\|x\|^{2} \geqslant\left\|T^{n} x\right\|^{2} \geqslant\left\|\left(T^{*} T\right)^{1 / 2} T^{n-1} x\right\|^{2} \\
& \quad=\left\|\left(T^{*} T\right)^{1 / 2} E T^{n-1} x+\left(T^{*} T\right)^{1 / 2}(I-E) T^{n-1} x\right\|^{2} \geqslant \varepsilon\left\|(I-E) T^{n-1} x\right\|^{2} .
\end{aligned}
$$

Hence $\left\|(I-E) T^{n-1}\right\| \leqslant \sqrt{\varepsilon}$. Now

$$
\begin{aligned}
(I-E) T^{n-1} & =[(I-E) T]^{n-1}+(I-E) T E T[(I-E) T]^{n-2} \\
+ & (I-E) T^{2} E T[(I-E) T]^{n-3}+\cdots+(I-E) T^{n-2} E T .
\end{aligned}
$$

Since every term on the right except the first has norm $\leqslant \sqrt{\varepsilon}$ we have that

$$
\left\|[(I-E) T(I-E)]^{n-1}\right\| \leqslant\left\|[(I-E) T]^{n-1}\right\| \leqslant(n-1) \sqrt{\varepsilon} .
$$

THEOREM 4. Let $p_{n}(T)=T^{n}$. Then the set of nilpotent operators of index $n$ is asymptotic for $p_{n}$.

Proof. The result is trivial for $n=1$ and meaningless for $n=0$. If $n=2$, define $E$ as in Lemma 1 . Then $\hat{T}=E T(I-E)$ is nilpotent of index two. We may assume $\|T\| \leqslant 1$. But $\|T-\hat{T}\|=\|(I-E) T+E T E\| \leqslant 2 \sqrt{\varepsilon}$ by the proof of Lemma 1 . Hence Theorem 4 holds for $n=2$. Suppose now that Theorem 4 holds for $n \leqslant k-1$. To see that it holds for $k$, suppose that $\left\|T^{k}\right\|<\varepsilon,\|T\| \leqslant 1$. Let $\delta_{n}(\varepsilon, K)$ be the $\delta$ of Definition 1 for $p_{n}$. Define $E$ as in Lemma 1. Then relative to $E \oplus(I-E)$ we have

$$
T=\left[\begin{array}{ll}
E_{1} & T_{1} \\
E_{2} & T_{2}
\end{array}\right] \text { with }\left\|\left[\begin{array}{ll}
E_{1} & 0 \\
E_{2} & 0
\end{array}\right]\right\|<\sqrt{\varepsilon} .
$$

By Lemma $1,\left\|T_{2}^{k-1}\right\| \leqslant(k-1) \sqrt{\varepsilon}$. Hence by setting $E_{1}=E_{2}=0$, and perturbing $T_{2}$ by $\delta_{n-1}((k-1) \sqrt{\varepsilon}, 1)$ we have a nilpotent of index at most $k$.

Corollary 1. Let $p_{n}(T)=T^{n}$, and $\delta_{n}(\varepsilon, 1)$ be the $\delta$ of Definition 1. Then $\delta_{2}(\varepsilon, 1)$ may be taken as $2 \sqrt{\varepsilon}$ and $\delta_{n}(\varepsilon, 1)$ as $\sqrt{\varepsilon}+\delta_{n-1}((n-1) \sqrt{\varepsilon}, 1)$.

We can now prove our main result.

THEOREM 5. Let $p(\lambda)$ be a polynomial. Let $\Delta=\{T: p(T)=0\}$. Then $\Delta$ is asymptotic for $p$.

Proof. Let $p(\lambda)=\left(\lambda-\lambda_{1}\right)^{m_{1}} \cdots\left(\lambda-\lambda_{r}\right)^{m_{1}}$. If $r=1$, Theorem 5 follows from Theorem 4. Assume that $r \geqslant 2$ and $\lambda_{1}, \ldots, \lambda_{r}$ are all distinct. There exists an $\varepsilon_{1}$ such that the $r$ circles of radius $\varepsilon_{1}$ centered at $\lambda_{1}, \ldots, \lambda_{r}$ have mutually disjoint interior. Let $\varepsilon_{0}$ be such that if $|p(\lambda)|<\varepsilon_{0}$, then $\lambda$ is contained in the interior of one of these circles. Let

$$
P_{k}=\frac{1}{2 \pi i} \int_{\left|\lambda-\lambda_{k}\right|=\varepsilon_{1}}(\lambda-T)^{-1} d \lambda .
$$

We shall first show that $\left\|P_{k}\right\|$ is bounded by a function of $\|p(T)\|,\|T\|, \varepsilon_{0}$, $p(\lambda)$ for small $\|p(T)\|$. Observe that $p(\lambda)-p(T)=(\lambda-T) q(\lambda, T)$ for a polynomial $q$ in the variables $\lambda, T$. Thus 


$$
I-p(T) p(\lambda)^{-1}=(\lambda-T) q(\lambda, T) p(\lambda)^{-1} .
$$

Now for $\lambda$ such that $\left|\lambda-\lambda_{k}\right|=\varepsilon_{1}$ we have $\|p(T) / p(\lambda)\|$ is small if $\|p(T)\|$ is. Let $E=p(T) / p(\lambda)$. Then

$$
(\lambda-T)^{-1}=q(\lambda, T) / p(\lambda)(I-E)^{-1}=q(\lambda, T) / p(\lambda)+J
$$

where $\|J\| \leqslant\|q(\lambda, T) / p(\lambda)\|\|E\| /(I-\|E\|)$ is small since $\|q(\lambda, T) / p(\lambda)\|$ is bounded by a function of $\|T\|$ and $p(\lambda)$. Thus the $P_{k}$ are bounded.

We now show that $\left(T-\lambda_{k}\right)^{m_{k}} P_{k}$ is small. From (1) we have

$$
\begin{aligned}
\left\|\left(T-\lambda_{k}\right)^{m_{k}} P_{k}\right\| & =\left\|\frac{1}{2 \pi i} \int_{\left|\lambda-\lambda_{k}\right|=\varepsilon_{1}} \frac{\left(\lambda-\lambda_{k}\right)^{m_{k}}}{p(\lambda)} q(\lambda, T)+\left(\lambda-\lambda_{i}\right)^{m_{k}} J d \lambda\right\| \\
& =\left\|\frac{1}{2 \pi i} \int_{\left|\lambda-\lambda_{k}\right|=\varepsilon_{1}}\left(\lambda-\lambda_{k}\right)^{m_{k}} J d \lambda\right\| \leqslant\|J\| \varepsilon_{0}^{m_{k}+1} .
\end{aligned}
$$

Since each $\left(T-\lambda_{k}\right)^{m_{k}} P_{k}$ is small we may use Theorem 4 to perturb $T P_{k}$ to get a $\hat{T}_{k}$ on $R\left(P_{k}\right)$ such that $\left(\hat{T}_{k}-\lambda_{k}\right)^{m_{k}} P_{k}=0$. Defining $\hat{T}=\sum_{k=1}^{r} \hat{T}_{k} P_{k}$ gives the required perturbation of $T$ such that $p(\hat{T})=0$.

5. Comments. We have intentionally avoided assuming anything about $T$ other than $\left\|p\left(T^{*}, T\right)\right\|<\varepsilon$. There are a large number of interesting questions of the form "Is $\Delta$ asymptotic for $p\left(T, T^{*}\right)$ for $T \in X$ ?" where $X$ is some class of operators. Note that the $T_{n}$ of Example 1 are hyponormal so that hyponormality is not enough to make the normals asymptotic for $p\left(T, T^{*}\right)=$ $T^{*} T-T T^{*}$.

There are several other classes of operators that are defined by polynomials. For example $(B N)=\left\{T: p\left(T, T^{*}\right)=\left[T^{*} T, T T^{*}\right]=0\right\}$. It is not known if $(B N)$ is asymptotic for $p$. Examples of operators in $(B N)$ may be found in [2].

Questions about whether a given class $\Delta$ is asymptotic for a given $p\left(T, T^{*}\right)$ can often be restated in terms of pairs of selfadjoint operators. For instance, Example 1 shows that there exist hermitian $A, B$ with norms close to one, such that $\|A B-B A\|$ is small but there do not exist hermitian $\hat{A}, \hat{B}$ close to $A, B$ respectively such that $\hat{A} \hat{B}-\hat{B} \hat{A}=0$.

One could define a somewhat stronger form of asymptotic behavior then we did by having $\delta(\varepsilon, K)$ depend on only $\varepsilon$. As to be expected, the weaker version sometimes holds when the stronger does not. For example an asymptotic version of Fuglede's Theorem holds when the sequence is uniformly bounded [7] but does not hold if the boundedness condition is omitted. Note that $\delta(\varepsilon, K)$ is independent of $K$ in Propositions 1-4. Also if $p(\lambda)=\lambda^{2}$, then one can take $\delta(\varepsilon, K)=\delta(\varepsilon)=2 \sqrt{\varepsilon}$. It would be of interest to know if $\delta(\varepsilon, K)$ can be taken independent of $K$ in Theorem 5 .

\section{REFERENCES}

1. J. Bastian and K. J. Harrison, Subnormal weighted shifts and asymptotic properties of normal operators, Proc. Amer. Math. Soc. 42 (1974), 475-479. 
2. S. L. Campbell, Linear operators for which $T^{*} T$ and $T T^{*}$ commute. II, Pacific J. Math. 53 (1974), 355-361.

3. Linear operators for which $T^{*} T$ and $T+T^{*}$ commute, Pacific J. Math. 61 (1975), 53-58.

4. S. L. Campbell and R. Gellar, Spectral properties of linear operators for which $T^{*} T$ and $T+T^{*}$ commute, Proc. Amer. Math. Soc. 60 (1976), 197-202.

5. L_ Linear operators for which $T^{*} T$ and $T+T^{*}$ commute. II, Trans. Amer. Math. Soc. 226 (1977), 305-319.

6. P. R. Halmos, Finite-dimensional noncommutative approximation theory, Talk at the 1973 Conference of Theoretical Matrix Theory, University of California, Santa Barbara, Calif.

7. R. Moore, An asymptotic Fuglede Theorem, Proc. Amer. Math. Soc. 50 (1975), 138-142.

Department of Mathematics, North Carolina State University, Raleigh, North Carolina 27607 